\title{
The influence of heating temperature and sizes of components upon stresses and defect formation in semiconductor structures under isothermal heating
}

\author{
O.A. Agueev, A.M. Svetlichny \\ Taganrog State University of Radio Engineering, GSP-17A, 44 Nekrasovsky Lane, Taganrog 347915, Russia, \\ E-mail: ageev@tsure.ru, tel: (863-44)6-16-11
}

\begin{abstract}
In the work a complex analysis of the influence of size of components and heating temperature upon stresses and defect formation in substrates of the $\mathrm{Si}_{-} \mathrm{SiO}_{2}$ structures is done. It is determined that alteration of shape and size of components can change stresses in the silicon substrate by an order, and the increase in heating temperature from 800 to $1100^{\circ} \mathrm{C}$ increases the defect formation criterion by two orders. The optimum size and shape alteration range as well as the heating temperature for defectfree thermal treatment of structures are determined.
\end{abstract}

Keywords: isothermal heating, thermo-elastic stresses, resultant shifting stresses.

Paper received 20.02.01; revised manuscript received 24.10.01; accepted for publication 12.12.01.

\section{Introduction}

Stresses in structures influence significantly an accepted field in microelectronics because of the irreversible electrophysical changes at the boundaries of IC components. The nature of excess stresses which lead to defect formation is rather complex and depends upon numerous factors: on substrates and films, concentration of impurities in the local alloyed areas, crystallographic orientation of a plate, heating and cooling conditions, size of IC components and other factors [1-4].

Although there are a great number of works investigating stresses and defect formation processes in IC structures, these problems require further investigation [1-6]. This is mainly connected with a complex and multilayer configuration of the IC structures, which contains a great number of different stress concentrators (film coating discontinuity, impurity, etc.). Thus, the aim of a stress state calculation in the actual IC structure is three-dimensional and extremely complicated to solve [1-8]. Nowadays the influence of heating temperature and size factors (size of a component, thickness of a film, size coefficient, which is determined by the relation of component side sizes) on stress fields being formed in the IC structure is insufficiently explored.
The aim of this work is to ascertain the regularities of size factors and temperature influence on stresses and defect formation in $\mathrm{Si}_{-} \mathrm{SiO}_{2}$ structures under isothermal heating.

\section{Stresses in semiconductor structures}

To achieve the above aim, it is necessary to calculate thermoelastic stresses in a typical component, which is most frequently found in the IC structures. One of these components is a window in a film (local alloying mask, windows in a dielectric layer for contacts, metal interconnections, etc.).

Setting the task one assumes that a substrate is an elastic body, which occupies a half-space $z \geq 0$. A film with thickness $\mathrm{h}$ contains discontinuity in the form of a window of $a \times b$ sizes. In this case, in accordance with [1], around film sides a concentration of stresses occurs in the film $\sigma_{f}$, which affect the substrate with the force of $\sigma_{f} \cdot h$. Thus, the substrate is a place for a cascade of elastic displacements, vector components $(u, v, w)$ of which can be calculated using the model [10].

Tensor deformation components are partial derivatives of displacement and are defined by the following expressions [11]: 


\section{O.A. Agueev, A.M. Svetlichny: The influence of heating temperature and sizes ...}

$\varepsilon_{x x}=\frac{\partial u}{\partial x} ; \varepsilon_{y y}=\frac{\partial v}{\partial y} ; \varepsilon_{z z}=\frac{\partial w}{\partial z} ; 2 \varepsilon_{x y}=\frac{\partial v}{\partial x}+\frac{\partial u}{\partial y}$

$2 \varepsilon_{y z}=\frac{\partial v}{\partial z}+\frac{\partial w}{\partial y} ; 2 \varepsilon_{z x}=\frac{\partial u}{\partial z}+\frac{\partial w}{\partial x}$.

For further analysis it is necessary to mention that in the substrate plane-stressed $(a>>h$ and $b>>h)$ and planestrained ( $a \sim h$ and $b \sim h$ ) conditions can be realized, each of which is characterized by three stress tensor components [11]:

- two normal ( $\sigma_{x x}$ and $\sigma_{y y}$ for plane-stressed condition, $\sigma_{x x}$ and $\sigma_{z z}$ for plane-strained condition)

- a tangent ( $\sigma_{x y}$ for plane-stressed condition, $\sigma_{x z}$ for plane-strained condition).

Hereinafter, to convert the expressions of one stressed condition to that of another, an index change $y y \leftrightarrow z z, x y \leftrightarrow x z$ should be done.

In accordance with the Hook generalized law for homogeneous isotropic body, expressions for these stress tensor components are of the following form [11]:

$$
\begin{aligned}
& \sigma_{x x}=\frac{E}{1+v}\left(\varepsilon_{x x}+\frac{v}{1-2 v}\left(\varepsilon_{x x}+\varepsilon_{y x}+\varepsilon_{z z}\right)\right) ; \\
& \sigma_{y y}=\frac{\mathrm{E}}{1+v}\left(\varepsilon_{y y}+\frac{v}{1-2 v}\left(\varepsilon_{x x}+\varepsilon_{y x}+\varepsilon_{z z}\right)\right) ; \\
& \sigma_{x y}=\frac{\mathrm{E}}{1+v} \varepsilon_{x y},
\end{aligned}
$$

where $E, v$ are Young's modules and Poisson's ratio for substrate material.

Table 1. Glide systems in the face-centered cubic crystal
When stresses exceed the Euler crippling stress $\left(\sigma_{E}\right)$, a plastic deformation takes place in solids, which is followed by a dislocation generation $[1,3]$. Here one should take into account that single-crystal substrates possess mechanical anisotropy, and to analyze defect formation one should calculate resultant shifting stresses - the stress tensor component projections on sliding systems corresponding crystallographic orientation. The calculation is performed in accord to the Schmidt law using the expressions [12]

$$
\begin{aligned}
& \tau_{s i}=\alpha_{x^{\prime} x} \alpha_{y^{\prime} x} \sigma_{x x}+\alpha_{x^{\prime} y} \alpha_{y^{\prime} y} \sigma_{y y}+ \\
& +\left(\alpha_{x x^{\prime}} \alpha_{y^{\prime} y}+\alpha_{x^{\prime} y} \alpha_{y x^{\prime}}\right) \sigma_{x y}
\end{aligned}
$$

where $\alpha_{x^{\prime} x}, \alpha_{y^{\prime} x}, \alpha_{x^{\prime} y}, \alpha_{y^{\prime} y}, \alpha_{x x^{\prime}}, \alpha_{y x^{\prime}}$ are aiming cosines between the directions corresponding indexes; $x$ is a normal vector to a film side (edge force line); $y$ is an axis perpendicular to axis $x$; $x^{\prime}$ is a sliding direction; $y^{\prime}$ is a normal vector to a glide plane.

For the plane-strained condition the expression (3) is of the form:

$$
\begin{aligned}
& \tau_{s i}=\alpha_{x^{\prime} x} \alpha_{z^{\prime} x} \sigma_{x x}+\alpha_{x^{\prime} z} \alpha_{z^{\prime} z} \sigma_{z z}+ \\
& +\left(\alpha_{x x^{\prime}} \alpha_{z^{\prime} z}+\alpha_{x^{\prime} z} \alpha_{z x^{\prime}}\right) \sigma_{x z}
\end{aligned}
$$

The signs are the same as in (3), with a change $y^{\prime} \leftrightarrow z^{\prime}$; $y \leftrightarrow z$.

To calculate resultant shifting stresses in substrates with a face-centered cubic lattice ( $\mathrm{Si}, 3 \mathrm{C}-\mathrm{SiC})$ one should take into consideration that glide planes are $\{111\}$ planes, and sliding directions are $<110>$. This corresponds to three sliding directions for each of four available sliding directions. If one assumes that substrate surface is oriented in direction (001), then analysis of the Thompson octahedron projection on the analyzed structure (Fig.2) gives 12 virtual sliding systems in the substrate (Table 1).

\begin{tabular}{lll}
\hline \hline Glide system & Glide direction & Glide planes \\
\hline \hline$\tau_{\mathrm{S} 1}$ & {$[1 \overline{1} 0]$} & $(111)$ \\
\hline$\tau_{\mathrm{S} 2}$ & {$[10 \overline{1}]$} & $(111)$ \\
\hline$\tau_{\mathrm{S} 3}$ & {$[01 \overline{1}]$} & $(111)$ \\
\hline$\tau_{\mathrm{S} 4}$ & {$[110]$} & $(1 \overline{1} 1)$ \\
\hline$\tau_{\mathrm{S} 5}$ & {$[10 \overline{1}]$} & $(1 \overline{1} 1)$ \\
\hline$\tau_{\mathrm{S} 6}$ & {$[0 \overline{1} 1]$} & $(111)$ \\
\hline$\tau_{\mathrm{S} 7}$ & {$[1 \overline{1} 0]$} & $(\overline{1} \overline{1} 1)$ \\
\hline$\tau_{\mathrm{S} 8}$ & {$[0 \overline{1} 1]$} & $(\overline{1} \overline{1} 1)$ \\
\hline$\tau_{\mathrm{S} 9}$ & {$[\overline{1} 0 \overline{1}]$} & $(\overline{1} \overline{1} 1)$ \\
\hline$\tau_{\mathrm{S} 10}$ & $(\overline{1} 11)$ \\
\hline$\tau_{\mathrm{S} 11}$ & $(111)$ \\
\hline$\tau_{\mathrm{S} 12}$ & {$[110]$} & $(\overline{1} 11)$ \\
\hline \hline
\end{tabular}




\section{O.A. Agueev, A.M. Svetlichny: The influence of heating temperature and sizes ...}

Calculating resultant shifting stresses for each of 12 sliding systems, one must take into account an edge force line, which is in agreement with a normal vector to a component edge (axis $x$ ) and the angle between sliding planes $\{111\}$ and plate plane (001), which makes $54^{\circ} 74^{\prime}$.

The results of resultant shifting stresses calculations in various sliding systems for plane-stressed and planestrained conditions are given in the Tables 2 and 3 , respectively.

According to the data given, resultant shifting stresses for examined structure can be combined into three groups:

$$
\begin{aligned}
& \tau_{1}=\tau_{S 1}=\tau_{S 4}=\tau_{S 7}=\tau_{S 10} \\
& \tau_{2}=\tau_{S 2}=\tau_{S 3}=\tau_{S 8}=\tau_{S 9} \\
& \tau_{3}=\tau_{S 5}=\tau_{S 6}=\tau_{S 11}=\tau_{S 12}
\end{aligned}
$$

The analysis shows that within a group resultant shifting stresses for the examined structure are symmetrically distributed and correlation between their absolute values is observed [9].

The Euler crippling stress depends on temperature and

\begin{tabular}{|c|c|c|}
\hline \multirow[t]{2}{*}{ Glide system } & \multicolumn{2}{|c|}{ Edge force direction } \\
\hline & 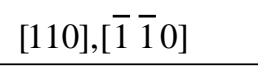 & {$[1 \overline{1} 0],[\overline{1} 10]$} \\
\hline$\tau_{S 1}$ & $\sqrt{\frac{2}{3}} \cdot \sigma_{x y}$ & $-\sqrt{\frac{2}{3}} \cdot \sigma_{x y}$ \\
\hline$\tau_{S 2}$ & $\frac{\sqrt{2}}{3}\left(\sigma_{x x}+\sigma_{x y}\right)$ & $\frac{\sqrt{2}}{3}\left(\sigma_{y y}-\sigma_{x y}\right)$ \\
\hline$\tau_{S 3}$ & $\frac{\sqrt{2}}{3}\left(\sigma_{x x}-\sigma_{x y}\right)$ & $\frac{\sqrt{2}}{3}\left(\sigma_{y y}+\sigma_{x y}\right)$ \\
\hline$\tau_{\mathrm{S4}}$ & $\sqrt{\frac{2}{3}} \cdot \sigma_{x y}$ & $-\sqrt{\frac{2}{3}} \cdot \sigma_{x y}$ \\
\hline$\tau_{S 5}$ & $\frac{\sqrt{2}}{3}\left(\sigma_{y y}+\sigma_{x y}\right)$ & $\frac{\sqrt{2}}{3}\left(\sigma_{x x}-\sigma_{x y}\right)$ \\
\hline$\tau_{56}$ & $\frac{\sqrt{2}}{3}\left(\sigma_{y y}-\sigma_{x y}\right)$ & $\frac{\sqrt{2}}{3}\left(\sigma_{x x}+\sigma_{x y}\right)$ \\
\hline$\tau_{S 7}$ & $-\sqrt{\frac{2}{3}} \cdot \sigma_{x y}$ & $\sqrt{\frac{2}{3}} \cdot \sigma_{x y}$ \\
\hline$\tau_{58}$ & $\frac{\sqrt{2}}{3}\left(\sigma_{x x}-\sigma_{x y}\right)$ & $\frac{\sqrt{2}}{3}\left(\sigma_{y y}+\sigma_{x y}\right)$ \\
\hline$\tau_{59}$ & $\frac{\sqrt{2}}{3}\left(\sigma_{x x}+\sigma_{x y}\right)$ & $\frac{\sqrt{2}}{3}\left(\sigma_{y y}-\sigma_{x y}\right)$ \\
\hline$\tau_{S 10}$ & $-\sqrt{\frac{2}{3}} \cdot \sigma_{x y}$ & $\sqrt{\frac{2}{3}} \cdot \sigma_{x y}$ \\
\hline$\tau_{S 11}$ & $\frac{\sqrt{2}}{3}\left(\sigma_{y y}+\sigma_{x y}\right)$ & $\frac{\sqrt{2}}{3}\left(\sigma_{x x}-\sigma_{x y}\right)$ \\
\hline$\tau_{S 12}$ & $\frac{\sqrt{2}}{3}\left(\sigma_{y y}-\sigma_{x y}\right)$ & $\frac{\sqrt{2}}{3}\left(\sigma_{x x}+\sigma_{x y}\right)$ \\
\hline
\end{tabular}
is defined by the expression [1]

Table 2. Resulting shear strains in the substrate characterized by a plane-stressed state 
O.A. Agueev, A.M. Svetlichny: The influence of heating temperature and sizes ...

Table 3. Resulting shear straines in the substrate characterized by the plane-deformed state

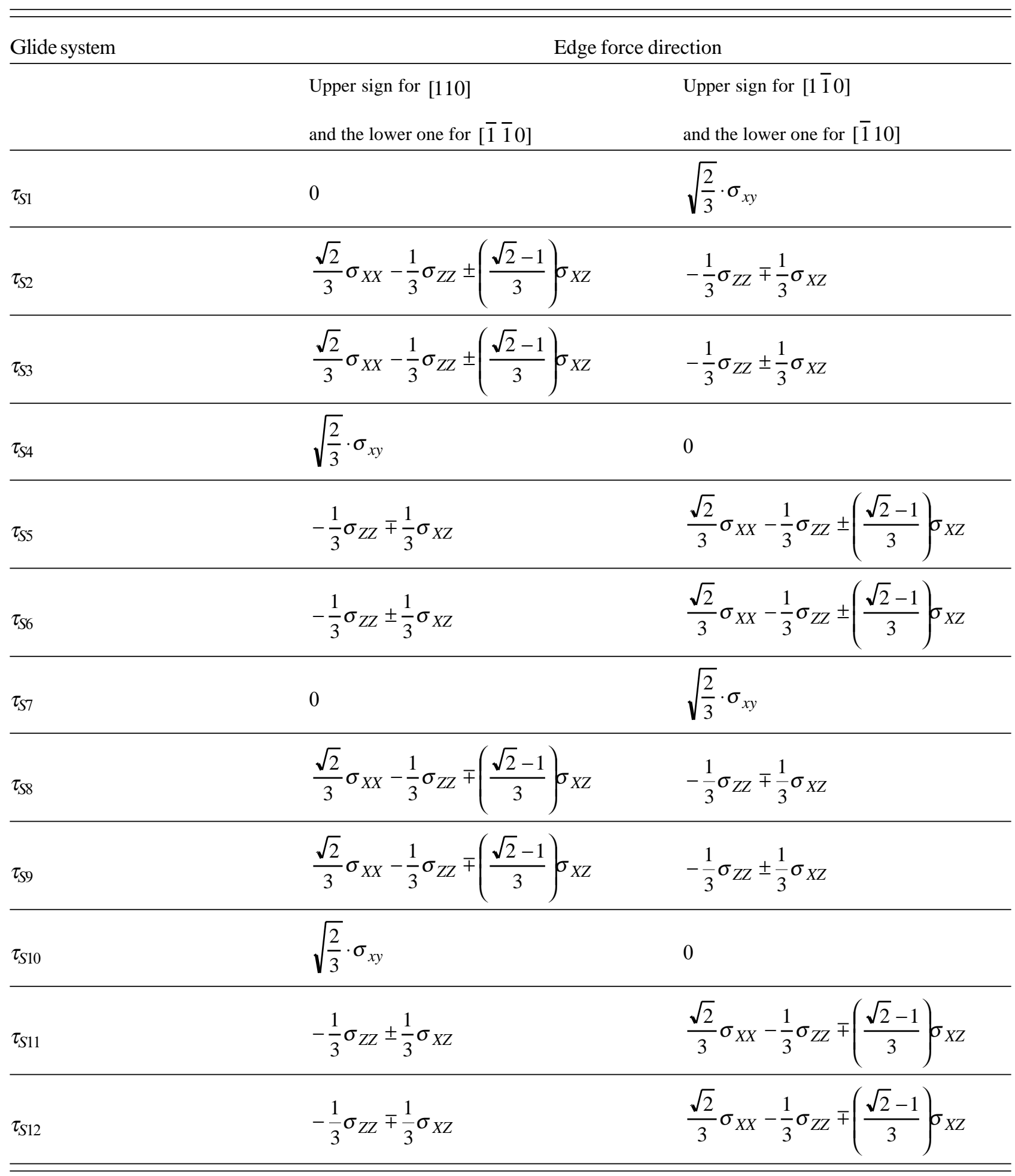

$$
\sigma_{E}(T)=H \cdot(\varepsilon)_{n}^{\frac{1}{n}} \exp \left(\frac{U}{n k T}\right)
$$

where $H=4.5 \cdot 10^{5}\left[\mathrm{dyne} / \mathrm{cm}^{2}\right]$ and $n=2.9$ are constants; $U=2.3[\mathrm{eV}]$ is plastic deformation activation energy; $\varepsilon=9 \cdot 10^{-5}\left[\mathrm{~s}^{-1}\right]$ is rate of deformation; $k=6.85 \cdot 10^{-5}[\mathrm{eV} / \mathrm{K}]$ is the Boltzmann constant [1].
Introducing defect formation criterion as $\tau$ to $\tau_{E}$ ratio, we can define conditions and areas of structure, where dislocation generation takes place [13].

Using the model for calculation of elastic shift vector components and expressions (1-5), one can evaluate resultant shifting stresses distribution in substrate with a window in dielectric film and through their comparison with 


\section{O.A. Agueev, A.M. Svetlichny: The influence of heating temperature and sizes ...}

the Euler crippling stress analyze the influence of size factors as well as heating temperature, on defect formation in substrates.

\section{Stresses in the $\mathrm{Si}_{-} \mathrm{SiO}_{2}$ structure}

As an example we will study a silicon substrate, where by thermal oxidation under $1100^{\circ} \mathrm{C}$ temperature $\mathrm{SiO}_{2}$ oxide layer of $h=1 \mu \mathrm{m}$ thickness was formed.

Stresses in the film $\sigma_{f}$ can be defined by such formula:

$\sigma_{f}=\sigma_{i n t}+\sigma_{t h}$

where $\sigma_{\text {int }}$ are stresses generated by formation, $\sigma_{t h}$ are thermoelastic stresses.

Stresses in the $\mathrm{SiO}_{2}$ film can be defined within the model given in [14] using $\mathrm{Si}$ and $\mathrm{SiO}_{2}$ physico-mechanical parameters [3]. In [13] stresses in the $\mathrm{SiO}_{2}$ film under heating to $800^{\circ} \mathrm{C}$ were determined: $\sigma_{i n t}=-2.10 \cdot 10^{9} \mathrm{din} / \mathrm{cm}^{2} ; \sigma_{t h}=$ $2.55 \cdot 10^{9} \mathrm{dyne} / \mathrm{cm}^{2} ; \sigma_{f}=4.5 \cdot 10^{8} \mathrm{dyne} / \mathrm{cm}^{2}$.

In this case, calculated according to the model given above, stress tensor component distributions $\sigma_{x x}, \sigma_{y y}, \sigma_{x y}$ in substrate with a film window is of the form represented in the Fig. 3. The calculation was performed in an area of $200 \times 200 \mu \mathrm{m}^{2}$ (the window dimension is $\mathrm{a} \times \mathrm{b}=100 \times 100 \mu \mathrm{m}^{2}$ ) with a $6.6 \mu \mathrm{m}$ step.

Analysis of the calculation results shows total correspondence to the regularities given in the works $[1,3$ $5,10,11]$ :

- stress tensor component concentration around window film boundaries;

- distribution symmetry in relation to the plane possessing window boundary and center of window;

- change of the normal stress tensor component character at the film boundary.

In the Fig. 4 calculation results of the stress tensor component maximum values for different size elements in dependence on a shape coefficient are represented. In the calculations a size coefficient changed from 0.1 to 10 , when $b / a<1 a=$ const was taken, and when $b / a>1 b=$ const was taken. The calculation was performed by such technique for $a \times b=100 \times 100 \mu \mathrm{m}^{2}$ and $a \times b=10 \times 10 \mu \mathrm{m}^{2}$ sized structures.

Analysis of relations shows that conversion from $100 \times 100 \mu \mathrm{m}^{2}$ sized components to $10 \times 10 \mu \mathrm{m}^{2}$ sized components changes stress tensor component maximum values by an order. Moreover, the change of size coefficient up to one order causes almost 10-fold increase in normal stress tensor component maximum values and weakly influences tangential components.

In the Fig. 5 represented are calculation results of the maximum value defect formation criterion dependence on an element shape coefficient for its different sizes. The calculation was performed by the same technique as the dependence calculations represented in the Fig. 4.

Analysis of the represented results shows that for $100 \times 100 \mu \mathrm{m}^{2}$ sized components, in the shape coefficient variation range studied, defect formation does not take place. Whereas for $10 \times 10 \mu^{2}$ sized components, if $0.25<b / a>4.0$, defect formation in substrates takes place. Furthermore, it is necessary to note, that $\tau_{2}$ when $b / a>1$ and $\tau_{3}$ when $b / a<$ 1 exert guiding influence on defect formation criterion maximum values, thus, hereafter, analyzing heating temperature influence we will study only these components.

Heating temperature variation influences an IC component stressed condition and changes crippling stress. In the Figs 6 and 7, represented are dependences of defect formation criterion maximum values calculated for different heating temperatures on shape coefficient for $100 \times 100 \mu \mathrm{m}^{2}$ and $10 \times 10 \mu \mathrm{m}^{2}$ sized components, respectively. Analysis of the data introduced shows that decrease in component sizes reduces the variation range of the shape coefficient and heating temperature that does not cause defect formation in substrates.

\section{Conclusions}

In the presented work, an analysis of resultant shifting stresses in face-centered cubic crystals under planestressed and plane-strained conditions is performed.

Moreover, a complex analysis of the influence of a typical IC component size factors and heating temperatures on stresses and defect formation in the $\mathrm{Si}-\mathrm{SiO}_{2}$ structure substrates is done. It is determined that size factors can change stresses in silicon substrates by one order, and heating temperature growth from 800 to $1100{ }^{\circ} \mathrm{C}$ increases defect formation criterion by two orders.

Optimum shape coefficient and heating temperature variation range for defect-free thermal treatment of structure are determined.

Analysis of the data obtained shows that optimizing technological regimes of thermal treatment one should take into account size factors. In case of component size decrease, stresses in substrates increase even under the same thermal treatment regimes.

The results introduced can be used in optimization of thermal treatment regimes when designing and manufacturing IC, as well as in researches of technological processes in microelectronics activated using laser, electronbeam and photon irradiation, as they allow to calculate modes of local dislocation arrangement generation [15].

\section{References}

1. S.M.Hu, Stress-related problem in silicon technology // $J$. Appl. Phys. 70, p. R53 (1991).

2. S.C. Jain, H.E. Maes, K. Pinardi, I. De Wolf, Stresses and strains in lattice-mismatches stripes, quantum wires, quantum dots, and substrates in Si technology // J. Appl. Phys. 79, p. 8145 (1996).

3. N.P. Zakharov, A.V. Bogdasaryan, Mechanical phenomene in IC. Moscow, Radio i svyaz', 1992 (in Russian).

4. V.S. Sergeev, O.A. Kuznetsov, V.A. Letyagin, Stresses and strains in IC elements. Moscow, Radio i svyaz', 1987 (in Russian).

5. J. Vanhellemont, S. Amelinckx, Film-edge-induced dislocation generation in silicon substrates // J. Appl. Phys. 61, p. 2170 (1987).

6. S. Mil'shtein, Dislocations in microelectronics // Phys. Stat. Sol. (a) 171, p. 371 (1999). 


\section{O.A. Agueev, A.M. Svetlichny: The influence of heating temperature and sizes ...}

7. R. Fournet, J.M. Salazar, Formation of dislocation patterns: computer simulation // Physical Review B. 53(10), p. 6283.

8. Debin Ren, Weiping Jia, Shouxin Li, Zhongguang Wang, Zhaoxing Peng, Finite element analysis elastic stress and the resolved shear stress in the primary slip system tricrystal // Phys. Stat. Sol. (a) 171, p. 443 (1999).

9. O.A. Agueev, A.M. Svetlichny, Effect of dimension factors of IC elements on stresses and defect formation in a substrate under isothermal heating (in Russian) // Izvestiya TRTU, №3, p. $21(2000)$

10. S. Isomae, Stress distributions in silicon crystal substrates with thin films // J. Appl. Phys. 54(4), p. 2782 (1981).

11. B. Boly, J. Weiner, Theory of temperature stresses. Moscow, Mir, 1964.
12. A. Fischer, Calculation of the shear stress in (111)- and (001) semiconductor substrates with film edges // Crystal Res.\&Technol. 20. p. 65 (1985).

13. O.A. Agueev, A.M. Svetlichny, Thermoelastic streses and defect production in semiconductor-insulator structures at isothermic heating // Semiconductor Physics, Quantum Electronics \& Optoelectronics, 3(3), p. 338 (2000).

14. H.C.Liu, S.P.Murarka, Elastic and viscoelastic analys of stress in thin films // J.Appl.Phys. 72, p. 3458 (1992).

15. F.D. Kasimov, D.A. Sechenov, F.G. Agayev, A.M. Svetlichny, O.A. Agueev, Activated processes in microelectronic technologies. Baku, ELM, 2000. 